Artículo

\title{
Proteínas trasportadoras de fósforo de la familia PHT1 y su uso potencial en la agricultura moderna
}

\author{
Juan Daniel Lira-Morales ${ }^{1}$ \\ Marino Valenzuela-López ${ }^{2}$ \\ María A. Islas-Osuna ${ }^{3}$ \\ Tomás Osuna-Enciso ${ }^{1}$ \\ José A. López-Valenzuela ${ }^{4}$ \\ Josefa Adriana Sañudo-Barajas ${ }^{1 \S}$
}

${ }^{1}$ Centro de Investigación en Alimentación y Desarrollo AC. Carretera Eldorado km 5.5, Col. Campo El Diez, Culiacán, Sinaloa, México. CP. 80110. (juan.lira@estudiantes.ciad.mx; osuna@ ciad.mx). ${ }^{2}$ Facultad de Agronomía-Universidad Autónoma de Sinaloa. Carretera Culiacán-Eldorado km 17.5, Culiacán, Sinaloa, México. CP. 80000. (marinova6@hotmail.com). ${ }^{3}$ Centro de Investigación en Alimentación y Desarrollo AC. Carretera Gustavo Enrique Astiazarán Rosas núm. 46, Col. La Victoria, Hermosillo, Sonora, México. CP. 83304. (islasosu@ ciad.mx). ${ }^{4}$ Facultad de Ciencias Químico Biológicas-Universidad Autónoma de Sinaloa. Culiacán, Sinaloa, México. (jalopezvla@uas.edu.mx).

${ }^{\S}$ Autora para correspondencia: adriana@ciad.mx.

\section{Resumen}

La agricultura se ha globalizado por sus modernos avances orientados a producir más y mejores alimentos bajo un modelo de protección ambiental. Esta práctica se realiza en suelos con diferentes cantidades de nutrientes disponibles y se basa en el uso de fuentes minerales externas para satisfacer la demanda del cultivo. El fósforo $(\mathrm{P})$ es un macroelemento que participa en funciones vitales de las plantas como la producción de adenosín trifosfato (ATP), formación de biomembranas y reacciones de señalización, entre otras. Las plantas utilizan estrategias fisiomorfológicas ante una deficiencia de $\mathrm{P}$ que se manifiestan en síntomas característicos como desarrollo deficiente, elongación de raíz, maduración precoz y reducción de la productividad del cultivo como consecuencia. Para mantener la homeostasis celular, las plantas inducen la sobreproducción de proteínas de membrana con función transportadoras de fosfato en los diferentes órganos. Estas proteínas pertenecen a la familia PHT1, presentan un transporte de tipo sin porte que facilita la introducción de fosfato inorgánico $(\mathrm{Pi})$ desde la rizosfera y permiten satisfacer la demanda biológica durante los procesos de señalización y energía. Estructuralmente estas proteínas son altamente conservadas en plantas (monocotiledóneas y dicotiledóneas) y se caracterizan por poseer 12 dominios transmembranales, un dominio conservado 2A0109 y tamaño aproximado de 520 aa. El objetivo de la presente revisión es situar en perspectiva el conocimiento actual de las proteínas transportadoras de fosfato PHT1, tomando de base los avances en modelos biológicos para mejorar los procesos productivos y las técnicas de manejo nutricional en los cultivos.

Palabras clave: agricultura, fertilizantes, fósforo, homeostasis, mejoramiento, PHT1.

Recibido: mayo de 2019

Aceptado: agosto de 2019 


\section{Introducción}

Algunos elementos minerales son esenciales para el crecimiento y desarrollo de las plantas, los cuales se pueden clasificar por su concentración en la planta en macronutrientes y micronutrientes (Agrios, 2004). La deficiencia de estos elementos altera el metabolismo y la fisiología de las plantas, con síntomas característicos según el elemento limitante. Los síntomas visuales de los desbalances nutricionales son considerados marcadores tardíos de la deficiencia; sin embargo, son precedidos por procesos moleculares de señalización temprana o de respuesta biológica intermedia que pueden servir de marcadores tempranos para identificar la deficiencia, generar estrategias de manejo y evitar las pérdidas productivas.

La magnitud de respuesta espacio-temporal de los desbalances nutricionales varía en función del mineral, por ejemplo; nitrógeno, $\mathrm{P}$ y potasio pueden traslocarse rápidamente y suplir la demanda metabólica-estructural mientras que elementos como boro, hierro y calcio, son de menor movilidad por lo que el impacto de su deficiencia difiere del resto (Taiz \& Zeiger, 2002). Las plantas de manera natural se pueden encontrar en condiciones de deficiencia, pero gracias a su variabilidad genética han sido capaces de modificar su fisiología y metabolismo con el fin de adaptarse y sobrevivir a estas condiciones (Ciereszko y Barbachowska, 2000).

Dentro de los principales mecanismos se encuentran la sobreexpresión de genes relacionados con las proteínas transportadoras PHT1, las cuales son reguladas de una manera diferencial según el órgano de la planta, el tiempo de exposición a la deficiencia y los niveles de nutrición de una manera compleja (Nagy et al., 2006). Dichas proteínas tienen una afinidad de transporte a fosfato que difiere entre especies, por los que es necesario profundizar en el conocimiento del funcionamiento y regulación de estas proteínas en diferentes organismos y modelos experimentales, para tener más herramientas disponibles para afrontar los retos venideros.

\section{Fertilizantes fosforados}

El cultivo intensivo de plantas implica necesariamente la fertilización con $\mathrm{P}$ a niveles que satisfagan los requerimientos específicos de cada etapa fenológica del ciclo productivo. La demanda de fertilizantes fosfatados se ha incrementado en los últimos años y se proyecta que en 2018 alcance los 46.6 millones de toneladas (FAO, 2015). El incremento anual estimado es de 4\% en América y 2.4\% a nivel mundial, poniendo en riesgo el abasto interno de los mismos (FAO, 2015). Una de las limitaciones de la agricultura moderna es la disponibilidad de roca fosfórica, una fuente no renovable a partir de la cual se producen los fertilizantes fosforados (Zapata y Roy, 2007). Por lo tanto, es necesario considerar su disponibilidad en el contexto de la agricultura presente y futura; además, evaluar nuevas estrategias para satisfacer la demanda creciente y ambientalmente responsable.

\section{El P y su importancia en la planta}

El P es asimilado por las plantas como fosfato, siendo el ortofosfato monovalente $\left(\mathrm{H}_{2} \mathrm{PO}_{4}\right)$ el que se encuentra en mayor cantidad. Una vez incorporado forma parte de la unión entre nucleósidos y ribonucleósidos, además está presente en fosfolípidos de membranas unido a moléculas de amino colina y fosfatidilcolina. En la célula la mayor parte del $\mathrm{P}$ permanece almacenado en su forma inorgánica (Pi), cuya liberación en el medio intracelular puede desencadenar la explosión respiratoria durante la maduración (Hawkesford et al., 2012). 
El Pi también puede ser empleado en la síntesis de fósfato de alta energía ((P)-(P)) o en forma de éster (C-(P)), en ambas formas es empleado en procesos metabólicos de biosíntesis y degradación en donde se requiere principalmente ATP (Por ejemplo, biosíntesis y degradación fosforilativa del almidón). En la señalización interviene en reacciones de fosforilación por adenosin difósfato (ADP), adenosin trifósfato (ATP) o guanidin trifosfato (GTP) donde se libera como pirofosfato inorgánico (PPi), que se emplea posteriormente por diversas enzimas (Hawkesford et al., 2012).

La variedad de síntomas que se presentan en una planta deficiente de Pi son diversos debido a su importancia en procesos biológicos vitales (Figura 1). En hojas se manifiestan puntos necróticos y un menor crecimiento de la planta. Algunas especies se tiñen color púrpura (síntesis de antocianinas) en varios órganos y en deficiencia severa las hojas llegan a tornarse azul grisáseas y las nervaduras se obscurecen (Berry, 2010).

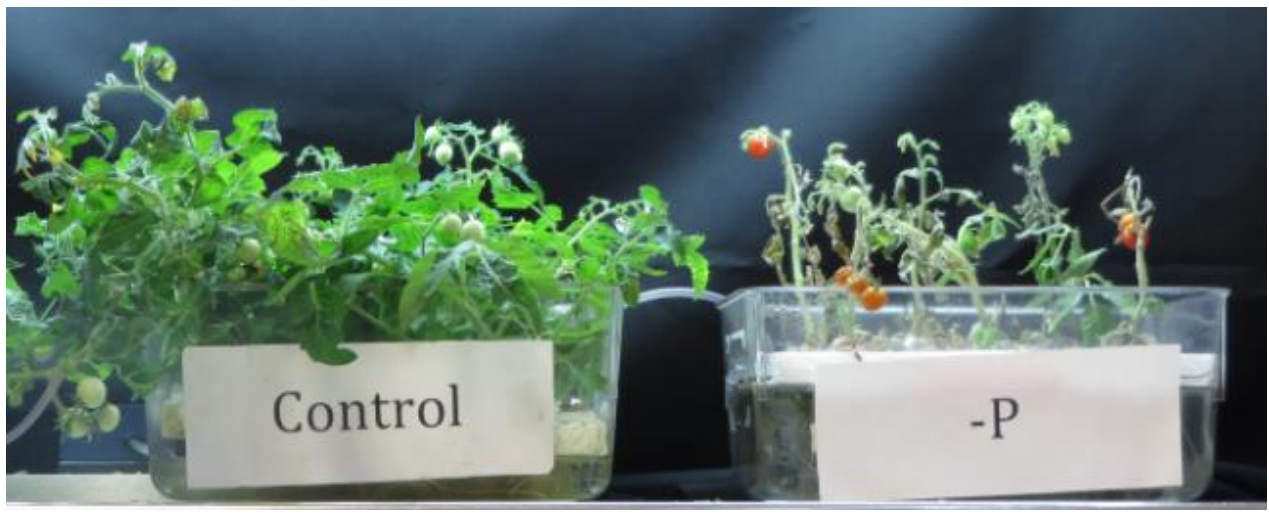

Figura 1. Plantas de tomate (Solanum lycopersicum cv Micro-tom) 60 días de tratamiento en condiciones de nutrición ideal (control) y deficiente de fósforo (-P). Semillas germinadas de tomate Micro-tom en medio nutritivo Murashige-Skoog (MS) a fotoperiodo de 16 h luz/8 h obscuridad $\left(27{ }^{\circ} \mathrm{C}\right.$ y $\sim 70 \%$ HR), después de tres semanas las plántulas se aclimataron en medio hidropónico con nutrición Steiner (testigo) durante tres semanas. Posteriormente las plantas se transfirieron a condiciones nutricionales diferenciales de $\mathrm{P}$ [bajo $\mathrm{P}$ (-P: $1.2 \mathrm{ppm})$ y control (P; 9 ppm)].

En maíz (Zea mays) y frijol (Phaseolus vulgaris), los síntomas incluyen un menor peso fresco, una disminución del contenido de $\mathrm{P}$ radicular, menor desarrollo en general de la planta y aumento de raíces laterales (Ciereszko et al., 1996; Hernández et al., 2007; Qui-Lun et al., 2007) en frijol además se acompaña de un cambio de color en hojas jóvenes (Hernández-Domíguez et al., 2012). No obstante, estas alteraciones fenotípicas ocurren de manera posterior y tardía como consecuencia de una serie de regulaciones génicas y bioquímicas.

A nivel bioquímico se manifiestan cambios en el metabolismo de carbohidratos para una rápida adaptación y recuperación a los niveles de Pi en la planta, entre ellos, la acumulación de azúcares (glucosa y almidón en hojas), sacarosa (en hojas y raíz) y de las enzimas fosfato sintasa, sacarosa sintasa e invertasas (Ciereszko y Barbachowska, 2000).

En maíz (Zea mays) también se presenta un incremento de las enzimas fosfatasa ácida, peroxidasa y superóxido dismutasa, posiblemente como estrategia de regulación del ambiente redox con lo cual contribuye a la adquisición de Pi y adaptación al estrés (Qui-Lun et al., 2007). Durante este 
adaptativo proceso también se ha observado en plantas de uva (Vitis vinifera) la acumulación de antocianinas, atribuido a la utilización de la fenilalanina en la ruta de síntesis de antocianinas y la reducción de su conversión en la síntesis proteica (Yin et al., 2012).

Estos cambios bioquímicos llegan a repercutir en las características fenotípicas de las plantas. En tomate, las estrategias adaptativas conocidas incluyen una mayor actividad de fosfatasa ácida asociada a un incremento de biomasa en raíz para mejorar la capacidad de liberación de la enzima (Goldstein et al., 1988a), también de enzimas tipo RNAasa, con actividad de fosfotransferasas (Nurnberger et al., 1990), la liberación de ambas enzimas es una estrategia de la planta para asimilar Pi del medio mediante la degradación de sustratos presentes en el área circundante a la rizosfera para su posterior absorción.

Es importante resaltar, que la fosfatasa ácida en tomate tiene dos variantes o isoformas, probablemente como mecanismo de regulación ya que éstas están compuestas de dos subunidades que además de mantener la actividad catalítica de manera independiente, poseen la capacidad de agregación, manteniendo la función dual como fosfatasa ácida y fosfatasa alcalina (Goldstein et al., 1988b).

Otro tipo de modificaciones de adaptación son las de tipo metabólico, mediante las cuales se activan rutas alternativas para mantener activo el ciclo glicolítico.

En Brassica nigra ante una deficiencia de fósforo, decrecen los niveles de Pi (fósfato inorgánico), ATP, ADP, Fru-2,6-P 2 (Fructosa 2,6-bifosfato) y PPi (pirofosfato inorgánico), mientras que la cantidad de aminoácidos libres se incrementa. Además, se incrementa la actividad de las enzimas PFP (PPi: D-fructosa 6-fosfato 1-fosfotransferasa) no fosforilante NAPD-G3PDH, PEP (fosfoenolpiruvato) fosfatasa y PEP (fosfoenolpiruvato) carboxilasa, para crear rutas alternativas al glicólisis (Duff et al., 1989). Estos cambios del metabolismo permiten a la planta adaptarse y sobrevivir a las condiciones de deficiencia de Pi.

\section{Aspectos moleculares de la deficiencia de $\mathbf{P}$}

La respuesta molecular a la deficiencia de Pi se presenta a nivel de órganos y organelos de manera diferente. Dentro de los factores fisiológicos resaltan la expresión de transportadores y factores de transcripción (Rubio et al., 2001; Nilsson et al., 2007), los primeros con la función de activar estrategias de captura y translocación del mineral hacia el interior de la célula, mientras que los segundos activan o reprimen cascadas de señalización y expresión génica.

La respuesta espacio-temporal es determinada por el potencial génico de la planta; en uva se manifiesta en las primeras 24 h y se orienta a la expresión de genes de la síntesis de antocianinas, entre ellos, chalcona sintasa, chalcona isomerasa, flavonona-3-hidroxilasa, dihidroflavonol-4reductasa, antocianin-sintetasa, UDP-glucosa, UDP-glucosa:flavonoide 3- $O$ glucosiltransferasa (UFGT) y un factor de transcripción VvmybA1 (Yin et al., 2012). Por otro lado, en frijol aproximadamente $13 \%$ de los genes expresados corresponden a proteínas transportadoras, aunque también se presentaron cambios en genes relacionados al metabolismo secundario, transducción de señales, defensa de estrés y ciclo celular, entre otros (Hernández et al., 2007). 
La respuesta global a una deficiencia de este elemento en otras especies como Arabidopsis thaliana involucra la expresión diferencial de 1835 genes durante las primeras $72 \mathrm{~h}$ de exposición al estrés mineral por $\mathrm{Pi}$ (Wu et al., 2003). En arroz (Oryza sativa), la deficiencia de Pi también produjo una regulación génica negativa con 12990 genes reprimidos (21\% de los genes evaluados), mientras que el total de genes activados fue de8043 (13\%) (Park et al., 2012).

Además, las proteínas más producidas por un estrés por deficiencia de $\mathrm{P}$ es el transportador de fósforo PHT1;4 y la fosfatasa ácida ACP5 (Lan et al., 2012) lo que corrobora la participación de estas proteínas en el proceso de adaptación de la planta a la deficiencia de P. En arroz (Oryza sativa), 7 días de deficiencia de $\mathrm{P}$ modularon la expresión de genes en raíz y tallo, activando la expresión de $\sim 450$ genes en raíz y $\sim 250$ en tallo. Por otro lado, reprimieron la expresión de $\sim 400$ genes en raíz y $\sim 180$ en tallo.

Es importante mencionar que durante los 7 días se observó la activación de genes que codifican para factores de transcripción en raíz, mientras que en tallo esto sucede hasta las 24 h posteriores del estímulo. Otro dato de relevancia es que en raíz la expresión de algunos genes miembros de la familia PHT1 constante durante la presencia del estímulo (Cai et al., 2012). Esto indica que el proceso ocurre en una coordinación espacio-temporal ante una deficiencia de $\mathrm{Pi}$, donde el primer evento de absorción desencadena la respuesta más temprana de captura del mineral en raíz y posteriormente la translocación y movilización del Pi que realizan otros miembros de la misma familia de genes.

A nivel proteómico en maíz (Zea mays) se evaluaron los genotipos 99038 (tolerante a bajas concentraciones de P) y Qi-319 (silvestre), observándose la producción 140 proteínas acumuladas diferencialmente ante una deficiencia de $\mathrm{Pi}$, dentro de las más reguladas se encuentran las relacionadas con el metabolismo de carbono y la proliferación celular (Li et al., 2008). Estos procesos son vitales en el desarrollo de la planta y requieren un suministro constante de Pi, donde el proceso de reasimilación de este elemento ante una deficiencia se encuentra ampliamente relacionado con la producción de proteínas transportadoras de Pi de la familia PHT1.

\section{Proteínas PHT1}

Las proteínas transportadoras de P de la familia PHT1 son importantes en el proceso de recuperación del homeostasis en las plantas. Estas proteínas son dependientes del factor de transcripción PHR1 (phosphate starvation response) que regula los genes phosphate starvation induced (PSI), por sus siglas en inglés, incluidos los genes de la familia PHT1 (Bari et al., 2006).

Estas proteínas contienen 12 dominios transmembranales con una longitud aproximada de 520 aa (Poirier y Bucher, 2002), presentan una secuencia única conservada GGDYPLSATIxSE entre todos sus miembros (Karandashov y Bucher, 2005) y su regulación transcripcional y postranscripcional es un proceso complejo que involucra a la ubiquinasa PHO2, el microARN miR399 y el factor de transcripción PHR1.

La producción de las proteínas PHT1 es regulada por la ubiquinasa PHO2, la cual en condiciones normales degrada las proteínas PHT1 de manera activa; sin embargo, los transcritos de PHO2 contienen en la región 5'-UTR una secuencia que es reconocida por el miR399 que al interactuar con esta región conduce a la degradación del transcrito, evitando la producción de la proteína, a su 
vez, miR399 es regulado positivamente por el factor de transcripción PHR1 el cual está relacionado con las deficiencias de Pi (Bari et al., 2006), generando así, un ciclo de regulación dependiente de los niveles de Pi intracelular.

\section{Familia PHT1 en monocotiledóneas}

Nagy et al. (2006) evaluaron la expresión de 5 miembros de la familia PHT1 en maíz (Zea mays) en condiciones de deficiencia de Pi. Los genes PHT1, 1 y PHT1, 4 se expresan en todos los órganos de la planta con niveles de expresión más altos en las hojas y la raíz. Mientras que PHT1, 2 se expresa principalmente en raíz, PHT1, 3 en órganos reproductivos en las anteras y PHT1, 6 en raíz (Nagy et al., 2006). En Serratia italica, se observó una sobreexpresión de los genes SiPHT1: 8, SiPHT1: 9 y SiPHT1: 10 en tallo, sin cambio aparente en hojas y raíz, después de 15 días de una deficiencia de $\mathrm{P}(10 \mu \mathrm{M})$; sin embargo, después de 31 días de la deficiencia se observó en hoja una mayor expresión de SiPHT1, 4, SiPHT1, 6, SiPHT1, 11 y SiPHT1, 12 todo lo anterior al compararlas con plantas mantenidas en condiciones control (Ceasar et al., 2014).

Los patrones de expresión de los genes anteriormente mencionados pueden interpretarse como una acción coordinada para evitar redundancia funcional entre las proteínas y actuar de manera coordinada para translocar P desde la raíz hasta diversos órganos de la planta (Cuadro1).

Cuadro 1. Genes transportadores de fósforo de la familia PHT1.

\begin{tabular}{|c|c|c|}
\hline Especie & Gen(es) & Referencia \\
\hline Arabidopsis thaliana & $\begin{array}{l}\text { ARAth; PHT1;1, PHT1;2, PHT1;3, } \\
\text { PHT1;4, PHT1;5, PHT1;6, PHT1;7, } \\
\text { PHT1;8; PHT1;9 }\end{array}$ & $\begin{array}{l}\text { Muchhal y Raghothama } \\
\text { (1999) }\end{array}$ \\
\hline Capsicum frutescens & CfPT1, CfPT2, CfPT3, CfPT4, CfPT5 & (Chen et al., 2007) \\
\hline Glycine $\max$ & $\begin{array}{l}\text { GmPT1, GmPT2, GmPT3, GmPT4, } \\
\text { GmPT5, GmPT6, GmPT7, GmPT8, } \\
\text { GmPT9, GmPT10, GmPT11, GmPT12, } \\
\text { GmPT13, GmPT14 }\end{array}$ & $\begin{array}{l}\text { (Wu et al., 2011; Qin et } \\
\text { al., 2012) }\end{array}$ \\
\hline Hordeum vulgare & $\begin{array}{l}\text { Horvu: PHT1:1, PHT1;2, PHT1;3, PHT1:4, } \\
\text { PHT1:6 }\end{array}$ & $\begin{array}{l}\text { (Rae et al., 2003; } \\
\text { Zvobgo et al., 2018) }\end{array}$ \\
\hline Lotus japonicus & LjPT3 & (Maeda et al., 2006) \\
\hline Lycium barbarum & $\begin{array}{l}\text { LbPT1, LbPT2, LbPT3, LbPT4, LbPT5, } \\
\text { LbPT7 }\end{array}$ & (Hu et al., 2017) \\
\hline Medicago truncatula & MtPT1, MtPT2, MtPT3, MtPT5 & $\begin{array}{l}\text { (Kai et al., 2002; Liu et } \\
\text { al., 2008) }\end{array}$ \\
\hline Nicotiana tabacum & NtPT1, NtPT2, NtPT3, NtPT4, NtPT5 & (Chen et al., 2007) \\
\hline Oryza sativa & $\begin{array}{l}\text { OsPT1, OsPT 2, OsPT 3, OsPT4, OsPT5, } \\
\text { OsPT6, OsPT7, OsPT8, OsPT9, OsPT10, } \\
\text { OsPT11, OsPT12 }\end{array}$ & $\begin{array}{l}\text { (Paszkowski et al., } \\
\text { 2002; Yan et al., 2014; } \\
\text { Ye et al., 2017) }\end{array}$ \\
\hline
\end{tabular}




\begin{tabular}{|c|c|c|}
\hline Especie & Gen(es) & Referencia \\
\hline Poncirus trifoliata & $\begin{array}{l}\text { Pt1PT1, Pt1PT2, Pt1PT3, Pt1PT4, Pt1PT5, } \\
\text { Pt1PT6, Pt1PT7 }\end{array}$ & (Shu et al., 2012) \\
\hline Populus simonii & $\begin{array}{l}\text { PtPHT1:1: PtPHT1:2: PtPHT1:3: } \\
\text { PtPHT1:4: PtPHT1:5: PtPHT1:6: } \\
\text { PtPHT1:7: PtPHT1:8: PtPHT1:9 } \\
\text { PtPHT1:10 PtPHT1:11 PtPHT1:12: } \\
\text { PtPHT1:13, PtPHT1:14 }\end{array}$ & (Zhang et al., 2016) \\
\hline Populus trichocarpa & $\begin{array}{l}\text { PtPT1, PtPT2, PtPT3, PtPT4, PtPT5, } \\
\text { PtPT6, PtPT7, PtPT8, PtPT9, PtPT10, } \\
\text { PtPT11, PtPT12 }\end{array}$ & $\begin{array}{l}\text { (Loth-Pereda et al., } \\
\text { 2011) }\end{array}$ \\
\hline Pteris vittat & PvPHT1 & (Ditusa et al., 2016) \\
\hline Setaria italica & $\begin{array}{l}\text { StPHT1;1, StPHT1;2, StPHT1;3, } \\
\text { StPHT1;4, StPHT1;5, StPHT1;6, } \\
\text { StPHT1;7, StPHT1;8, StPHT1;9, } \\
\text { StPHT1;10, StPHT1;11, StPHT1;12 }\end{array}$ & (Ceasar et al., 2014) \\
\hline $\begin{array}{l}\text { Solanum } \\
\text { lycopersicum }\end{array}$ & $\begin{array}{l}\text { LePT1, LePT2, LePT3, LePT4, LePT5, } \\
\text { LePT6, LePT7, LePT8 }\end{array}$ & $\begin{array}{l}\text { (Liu et al., 1998; Nagy } \\
\text { et al., 2005; Chen et al., } \\
\text { 2014) }\end{array}$ \\
\hline Solanum melongena & SmPT1, SmPT2, SmPT3, SmPT4, SmPT5 & (Chen et al., 2007) \\
\hline Solanum tuberosum & $\begin{array}{l}\text { StPHT1:1, StPHT1:2, StPHT1:3, } \\
\text { StPHT1:4, StPHT1:5, StPHT1:6, } \\
\text { StPHT1:7, StPHT1:8 }\end{array}$ & $\begin{array}{l}\text { (Leggewie et al., 1997; } \\
\text { Nagy et al., 2005; Liu et } \\
\text { al., 2017) }\end{array}$ \\
\hline Sorghum bicolor & $\begin{array}{l}\text { SbPHT1:1, SbPHT1:2, SbPHT1:3, } \\
\text { SbPHT1:4, SbPHT1:5, SbPHT1:6, } \\
\text { SbPHT1:7, SbPHT1:8, SbPHT1:9 }\end{array}$ & $\begin{array}{l}\text { (Tavares De Oliveira } \\
\text { Melo, 2016) }\end{array}$ \\
\hline Triticum aestivum & $\begin{array}{l}\text { TaPHT1.1, TaPHT1.2, TaPHT1.6, } \\
\text { TaPHT1.8, TaPHT1.9, TaPHT1.10 }\end{array}$ & (Teng et al., 2017) \\
\hline Zea mays & $\begin{array}{l}\text { ZEAma: PHT1;1, PHT1;2, PHT1;3, } \\
\text { PHT1;4, PHT1;5, PHT1;6, PHT1;7, } \\
\text { PHT1;8, PHT1;9, PHT1;10, PHT1;11, } \\
\text { PHT1;12, PHT1;13 }\end{array}$ & $\begin{array}{l}\text { (Nagy et al., 2006; Liu } \\
\text { et al., 2016) }\end{array}$ \\
\hline
\end{tabular}

\section{Familia PHT1 en dicotiledóneas}

La familia PHT1 se ha identificado en varias especies y en Arabidopsis thaliana se compone de nueve genes con nomenclatura de PHT1, 1 a PHT1, 9 y su expresión es espacio-temporal específica por lo cual su papel fisiológico puede ser de alta regulación (Mudge et al., 2002). En Arabidopsis la represión de los genes PHT1, 1 y PHT1, 4 limita la absorción del mineral; su regulación es a nivel transcripcional y son un mecanismo de adaptación para la producción de proteínas de membrana transportadoras de P (Shin et al., 2004). La distribución tisular del gen PHT1, 4 es variada y su expresión depende de los órganos con disponibilidad de P (Misson et al., 2004). 
En tomate, la familia PHT1 está conformada por 8 miembros, LePT1 a LePT8, en este cultivo la presencia de LePT1 se identificó con una inducción temprana por deficiencia de $\mathrm{P}$ (en las primeras 24 h) (Muchhal y Raghothama, 1999). Chen et al. (2014) confirmaron que genes de la familia PHT1 están asociados a la deficiencia de P, LePT1 se expresó en raíz, tallo, hojas jóvenes, flores y frutos en los estados de madurez verde y maduro, pero la mayor expresión ocurrió en raíz. LePT2 solo fue detectado en raíz con una inducción $~ 15$ veces más en plantas con deficiencia de Pi respecto al control, LePT3, LePT5 y LePT7 se expresaron en todos los tejidos, pero sus mayores niveles se observaron en hoja, fruto maduro y raíz, respectivamente. LePT6 se expresó en raíz, tallo y hoja, pero en mayor medida en raíz.

Lo anterior sugiere que el transporte y asimilación de $\mathrm{P}$ en tomate y otras especies es un proceso altamente regulado y que existen variantes de espacio y tiempo que lo definen. En otras especies también de monocotiledóneas y dicotiledóneas se han caracterizado otros miembros de la familia PHT1 (Cuadro 1). En el cultivo de soya (Glycine max) se evaluó la expresión de 14 genes PHT1 bajo condiciones bajas de $\mathrm{P}(5 \mu \mathrm{M})$. Los mayores niveles de expresión en raíz los presentaron GmPT1, GmPT2, GmPT3, GmPT4, GmPT7, GmPT8, GmPT9 y GmPT12, en hoja GmPT13, en flor GmPT5 y en tallo GmPT6, GmPT9, GmPT11 y GmPT14; GmPT10 no presentó cambios en su expresión relativa durante la deficiencia de $\mathrm{P}(\mathrm{Wu}$ et al., 2011).

En el cuadro 1 se observa el avance sobre el conocimiento de las proteínas PHT1 transportadoras de Pi; sin embargo, en cuanto a los avances en el conocimiento de la regulación del metabolismo de $\mathrm{P}$, se han generado algunos modelos funcionales con base en estudios realizados en levaduras mutantes deficientes en asimilación de fósforo; por ejemplo, en las levaduras MB192 (Qin et al., 2012; Leggewie et al., 1997), PAM2 (Nagy et al., 2005) y EY917 (Nagy et al., 2006) se estudia el potencial del mejoramiento genético de las proteínas de interés en cultivos agrícolas importantes, mediante la evaluación de parámetros como la cinética de transporte de $\mathrm{P}$ y su afinidad por este mineral (Qin et al., 2012), esta información permitiría seleccionar genes con características deseables para el mejoramiento mediante ingeniería genética, con el fin de producir material más resistente a la deficiencia mineral, considerando los panoramas previstos ante la escasez prevista en los próximo años de los fertilizantes fosforados.

\section{Uso potencial como herramientas de mejoramiento}

La caracterización de las proteínas transportadoras de Pi sienta las bases para futuras aplicaciones. Este conocimiento podría llevar a crear plantas más adaptables a condiciones ambientales adversas como la deficiencia mineral mediante el uso de estrategias moleculares que permitirían obtener plantas más resilientes durante las primeras etapas de desarrollo, así como, a lo largo de su ciclo de vida. Otra alternativa es el uso de técnicas moleculares; por ejemplo, Yan et al. (2014), insertaron el gen OsPT6 de arroz en plantas de soya (NY-1001) y obtuvieron plantas más altas, con una mayor cobertura foliar, raíces más largas, un mayor contenido de $\mathrm{P}$, un crecimiento general más vigoroso y un aumento general en la biomasa.

Estos cambios fenotípicos se relacionaron con un incremento de al menos 5 veces en la expresión del gen OsPT6 en las plantas transformadas con respecto al control. Tales características fenotípicas son deseables para un cultivo intensivo debido a que se incrementan rasgos favorables relacionados con la productividad y resistencia a otros tipos de estrés bióticos o abióticos. Además de las secuencias codificantes, también se podrían explorar las regiones promotoras de genes de 
interés con la finalidad de incrementar la especificidad de la expresión en regiones localizadas de la planta para satisfacer una demanda en particular, por ejemplo, una mayor captación de $\mathrm{P}$ en suelos pobres en este elemento o en fructificación.

En Hordeum vulgare se observó que algunas regiones promotoras específicas presentan afinidad por la expresión en raíz, lo cual tiene potencial como herramienta de expresión para usar con genes de transportadores de minerales o codificantes para otras proteínas que sean necesarias en este órgano de la planta (Schünmann et al., 2004).

\section{Conclusiones}

Es factible la aplicación de estrategias conjuntas para mejorar el panorama futuro de la agricultura, tales como el mejoramiento genético y el manejo de la nutrición basado en indicadores de desbalances. Ambas requieren de conocimiento a profundidad de los mecanismos de regulación transcripcional, postranscripcional y postraduccional de las proteínas blanco dentro de los procesos de crecimiento y desarrollo. La estrategia de mejoramiento molecular es promisoria, en el caso de las proteínas PHT1 transportadoras de fósforo; sin embargo, los progresos son insuficientes y se requieren mayores esfuerzos para conocer su diversidad y características de regulación.

\section{Agradecimientos}

Al Consejo Nacional de Ciencia y Tecnología (CONACYT) por su apoyo para la realización de este trabajo.

\section{Literatura citada}

Agrios, G. 2004. Plant pathology. Elsevier Academic Press. Fifth Edition. San Diego, California. $922 \mathrm{p}$.

Bari, R.; Datt, P. B.; Stitt, M. and Scheible, W. R. 2006. PHO2, microRNA399, and PHR1 define a phosphate-signaling pathway in plants. Plant Physiol. 141(3):988-999.

Berry, W. 2010. Plant physiology online. Symptoms of deficiency in essential minerals. http://5e.plantphys.net/article.php?ch=5\&id=289.

Cai, H.; Xie, W.; Zhu, T. and Lian, X. 2012. Transcriptome response to phosphorus starvation in rice. Acta Physiologiae Plantarum. 34(1):327-341.

Ceasar, S. A.; Hodge, A.; Baker, A. and Baldwin, S. A. 2014. Phosphate concentration and arbuscular mycorrhizal colonisation influence the growth, yield and expression of twelve PHT1 family phosphate transporters in foxtail millet (Setaria italica). PLoS One. e108459. 9(9):1-12.

Chen, A.; Chen, X.; Wang, H.; Liao, D.; Gu, M.; Qu, H.; Sun, S. and Xu, G. 2014. Genome-wide investigation and expression analysis suggest diverse roles and genetic redundancy of PHT1 family genes in response to Pi deficiency in tomato. BMC Plant Biol. 14(61):1-15.

Chen, A.; Hu. J.; Sun, S. and Xu, G. 2007. Conservation and divergence of both phosphate and mycorrhiza-regulated physiological responses and expression patterns of phosphate transporters in solanaceous species. New Phytologist. 173(4):817-831. 
Ciereszko, I. and Barbachowska, A. 2000. Sucrose metabolism in leaves and roots of bean (Phaseolus vulgaris L.) during phosphate deficiency. J. Plant Physiol. 156(5-6):640-644.

Ciereszko, I.; Gniazdowska, A.; Mikulska, M. and Rychter, A. M. 1996. Assimilate translocation in bean plants (Phaseolus vulgaris L.) during phosphate deficiency. Plant Physiol. 149(34):343-348.

Ditusa, S. F.; Fontenot, E. B.; Wallace, R. W.; Silvers, M. A.; Steele, T. N.; Elnagar, A. H.; Dearman, K. M. and Smith, A. P. 2016. A member of the phosphate transporter 1 (PHT1) family from the arsenic-hyperaccumulating fern Pteris vittata is a high-affinity arsenate transporter. New Phytol. 209(2):762-72.

Duff, S.; Moorhead, G.; Lefebvre, D. and Plaxton, W. 1989. Phosphate starvation inducible 'bypasses' of adenylate and phosphate dependent glycolytic enzymes in Brassica nigra suspension cells. Plant Physiol. 90(4):1275-1278.

FAO (Food and Agriculture Organization). 2015. World fertilizer trends and outlook to 2018. https://www.agrilinks.org/library/world-fertilizer-trends-and-outlook-2018.

Goldstein, A and Baertlein, D. R. M. 1988a. Phosphate starvation inducible metabolism in Lycopersicon esculentum L.: excretion of acid phosphatase by tomato plants and suspension-cultured cells. Plant Physiol. 87(3):711-715.

Goldstein, A.; Danon, A.; Baertlein, D. and Mcdaniel, R. 1988b. Phosphate starvation inducible metabolism in Lycopersicon esculentum II: characterization of the phosphate starvation inducible-excreted acid phophatase. Plant Physiol. 87(3):716-720.

Hawkesford, M.; Horst, W.; Kichey, T.; Lambers, H.; Schjoerring, J.; Møller, I. S. and White, P. 2012. Chapter 6 - functions of macronutrients A2 - Marschner, Petra. In: Marschner's mineral nutrition of higher plants Third (Ed.). San Diego: Academic Press. 135-189 pp.

Hernández, G.; Ramírez, M.; Valdés-López, O.; Tesfaye, M.; Graham, M. A.; Czechowski, T.; Schlereth, A.; Wandrey, M.; Erban, A.; Cheung, F.; Wu, H. C.; Lara, M.; Town, C. D.; Kopka, J.; Udvardi, M. K. and Vance, C. P. 2007. Phosphorus stress in common bean: root transcript and metabolic responses. Plant Physiol. 144:752-767.

Hernández-Domíguez, E. E.; Valencia-Turcotte, L. G. and Rodríguez-Sotres, R. 2012. Changes in expression of soluble inorganic pyrophosphatases of Phaseolus vulgaris under phosphate starvation. Plant Sci. 187(2): 39-48.

Hu, W.; Zhang, H.; Zhang, X.; Chen, H. and Tang, M. 2017. Characterization of six PHT1 members in Lycium barbarum and their response to arbuscular mycorrhiza and water stress. Tree Physiology. 37(3):351-366.

Kai, M.; Takazumi, K.; Adachi, H.; Wasaki, J.; Shinano, T. and Osaki, M. 2002. Cloning and characterization of four phosphate transporter cDNAs in tobacco. Plant Sci. 163(4):837-846.

Karandashov, V. and Bucher, M. 2005. Symbiotic phosphate transport in arbuscular mycorrhizas. Trends in Plant Science. 10(1):22-29.

Lan, P.; Li, W. and Schmidt, W. 2012. Complementary proteome and transcriptome profiling in phosphate-deficient arabidopsis roots reveals multiple levels of gene regulation. Mol. Cel. Proteomics. 11(11):1156-1166.

Leggewie, G.; Willmitzer, L. and Riesmeier, J. W. 1997. Two cDNAs from potato are able to complement a phosphate uptake-deficient yeast mutant: identification of phosphate transporters from higher plants. Plant Cell. 9(3):381-392.

Li, K.; Xu, C.; Li, Z.; Zhang, K.; Yang, A. and Zhang, J. 2008. Comparative proteome analyses of phosphorus responses in maize (Zea mays L.) roots of wild-type and a low-P-tolerant mutant reveal root characteristics associated with phosphorus efficiency. Plant J. 55(6):927-939. 
Liu, B.; Zhao, S.; Wu, X.; Wang, X.; Nan, Y.; Wang, D. and Chen, Q. 2017. Identification and characterization of phosphate transporter genes in potato. J. Biotechnol. 264:17-28.

Liu, C.; Muchhal, U. S.; Uthappa, M.; Kononowicz, A. K. and Raghothama, K. G. 1998. Tomato phosphate transporter genes are differentially regulated in plant tissues by phosphorus. Plant Physiol. 116(1):91-99.

Liu, F.; Xu, Y.; Jiang, H.; Jiang, C.; Du, Y.; Gong, C.; Wang, W.; Zhu, S.; Han, G. and Cheng, B. 2016. Systematic identification, evolution and expression analysis of the Zea mays PHT1 gene family reveals several new members involved in root colonization by arbuscular mycorrhizal fungi. Int. J. Mol. Sci. 17(6):1-18.

Liu, J.; Versaw, W. K.; Pumplin, N.; Gomez, S. K.; Blaylock, L. A. and Harrison, M. J. 2008. Closely related members of the medicago truncatula PHT1 phosphate transporter gene family encode phosphate transporters with distinct biochemical activities. J. Biol. Chem. 283(36):24673-24681.

Loth-Pereda, V.; Orsini, E.; Courty, P-E.; Lota, F.; Kohler, A.; Diss, L.; Blaudez, D.; Chalot, M.; Nehls, U.; Bucher, M. and Martin, F. 2011. Structure and expression profile of the phosphate PHT1 transporter gene family in mycorrhizal <em>populus trichocarpa</em>. Plant Physiol. 156(4):2141-2154.

Maeda, D.; Ashida, K.; Iguchi, K.; Chechetka, S. A.; Hijikata, A.; Okusako, Y.; Deguchi, Y.; Izui, K. and Hata, S. 2006. Knockdown of an arbuscular mycorrhiza-inducible phosphate transporter gene of Lotus japonicus suppresses mutualistic symbiosis. Plant Cell Physiol. 47(7):807-17.

Misson, J.; Thibaud, M.; Bechtold, N.; Raghothama, K. and Nassaume, L. 2004. Transcriptional regulation and functional properties ofArabidopsis PHT1;4, a high affinity transporter contributing greatly to phosphate uptake in phosphate deprived plants. Plant Mol. Biol. 55(5):727-741.

Muchhal, U. and Raghothama, K. 1999. Transcriptional regulation of plant phosphate transporters. Proc. Natl. Acad. Sci. 96(10):5868-5872.

Mudge, S.; Rae, A.; Diatloff, E. and Smith, W. 2002. Expression analysis suggests novel roles for members of the PHT1 family of phosphate transporters inArabidopsis. Plant J. 31(3):341-353.

Nagy, R.; Karandashov, V.; Chague, V.; Kalinkevich, K.; Tamasloukht, M.; Xu, G.; Jakobsen, I.; Levy, A. A.; Amrhein, N. and Bucher, M. 2005. The characterization of novel mycorrhiza-specific phosphate transporters from Lycopersicon esculentum and Solanum tuberosum uncovers functional redundancy in symbiotic phosphate transport in solanaceous species. Plant J. 42(2):236-50.

Nagy, R.; Vasconcelos, M. J.; Zhao, S.; Mcelver, J.; Bruce, W.; Amrhein, N.; Raghothama, K. G. and Bucher, M. 2006. Differential regulation of five PHT1 phosphate transporters from maize (Zea mays L.). Plant Biol (Stuttg). 8(2):186-197.

Nilsson, L.; Muller, R. and Nielsen, T. H. 2007. Increased expression of the MYB-related transcription factor, PHR1, leads to enhanced phosphate uptake in Arabidopsis thaliana. Plant Cell Environ. 30(12):1499-1512.

Nurnberger, T.; Abel, S.; Jost, W. and Glund, K. 1990. Induction of an extracellular ribonuclease in cultured tomato cells upon phosphate starvation. Plant Physiol. 92(4):970-976.

Park, M. R.; Baek, S. H.; De Los Reyes, B. G.; Yun, S. J. and Hasenstein, K. H. 2012. Transcriptome profiling characterizes phosphate deficiency effects on carbohydrate metabolism in rice leaves. J Plant Physiol. 169(2):193-205. 
Paszkowski, U.; Kroken, S.; Roux, C. and Briggs, S. P. 2002. Rice phosphate transporters include an evolutionarily divergent gene specifically activated in arbuscular mycorrhizal symbiosis. Proceedings of the National Academy of Sciences. 99(20):13324-13329.

Poirier, Y. and Bucher, M. 2002 Phosphate transport and homeostasis in Arabidopsis. Arabidopsis Book. 1: e0024. doi:10.1199/tab.0024.

Qin, L.; Guo, Y.; Chen, L.; Liang, R.; Gu, M.; Xu, G.; Zhao, J.; Walk, T. and Liao, H. 2012. Functional characterization of 14 PHT1 family genes in yeast and their expressions in response to nutrient starvation in soybean. PLoS One. 7(10):1-13.

Qui-Lun, Y.; Ke-Cheng, Y.; Guang-Tang, P. and Thing-Zhao, R. 2007. The effects of low phosphorus stress on morphological and physiological characteristics of maize (Zea mays L.) Landrace. Agric. Sci. China. 6(5):559-566.

Rae, A. L.; Cybinski, D. H.; Jarmey, J. M. and Smith, F. W. 2003. Characterization of two phosphate transporters from barley; evidence for diverse function and kinetic properties among members of the PHT1 family. Plant Mol. Biol. 53(1):27-36.

Rubio, V.; Linhares, F.; Solano, R.; Martin, A. C.; Iglesias, J.; Leyva, A. and Paz-Ares, J. 2001. A conserved MYB transcription factor involved in phosphate starvation signaling both in vascular plants and in unicellular algae. Genes Dev. 15(16):2122-2133.

Schünmann; P. H. D.; Richardson, A. E.; Smith, F. W. and Delhaize, E. 2004. Characterization of promoter expression patterns derived from the PHT1 phosphate transporter genes of barley (Hordeum vulgare L.). J. Exp. Bot. 55(398):855-865.

Shin, H.; Shin, H. S.; Dewbre, G. R. and Harrison, M. J. 2004. Phosphate transport in Arabidopsis: PHT1;1 and PHT1;4 play a major role in phosphate acquisition from both low and high-phosphate environments. Plant J. 39(4):629-642.

Shu, B.; Xia, R-X. and Wang, P. 2012. Differential regulation of PHT1 phosphate transporters from trifoliate orange (Poncirus trifoliata L. Raf) seedlings. Sci. Hortic. 146:115-123.

Taiz, L. and Zeiger, E. 2002. Plant physiology. Sunderland: Sinauer Associates Third edition. $623 \mathrm{p}$.

Tavares, De Oliveira, M. A. 2016. Exploring genomic databases for in silico discovery of PHT1 genes in high syntenic close related grass species with focus in sugarcane (Saccharum spp.). Current Plant Biology. 6:11-18.

Teng, W.; Zhao, Y-Y.; Zhao, X-Q.; He, X.; Ma, W-Y.; Deng, Y.; Chen, X-P. adn Tong, Y-P. 2017. Genome-wide identification, characterization, and expression analysis of PHT1 phosphate transporters in wheat. Front Plant Sci. 8:1-14.

Wu, P.; Ma, L.; Hou, X.; Wang, M.; Wu, Y.; Liu, F. and Wang, X. 2003. Phosphate starvation triggers distinct alterations of genome expresion in Arabidopsis roots and leaves. Plant Physiol. 13(3):1260-1271.

Wu, Z.; Zhao, J.; Gao, R.; Hu, G.; Gai, J.; Xu, G. and Xing, H. 2011. Molecular cloning, characterization and expression analysis of two members of the PHT1 family of phosphate transporters in Glycine max. PLoS One. 6(6):1-12.

Yan, W.; Chen, G-H.; Yang, L-F.; Gai, J-Y. and Zhu, Y-L. 2014. Overexpression of the rice phosphate transporter gene OsPT6 enhances tolerance to low phosphorus stress in vegetable soybean. Sci. Hortic. 177:71-76.

Ye, Y.; Li, P.; Xu, T.; Zeng, L.; Cheng, D.; Yang, M.; Luo, J. and Lian, X. 2017. OsPT4 contributes to arsenate uptake and transport in rice. Front Plant Sci. 8:2197.

Yin, Y.; Borges, G.; Sakuta, M.; Crozier, A. and Ashihara, H. 2012. Effect of phosphate deficiency on the content and biosynthesis of anthocyanins and the expression of related genes in suspension-cultured grape (Vitis sp.) cells. Plant Physiol. Biochem. 55:77-84. 
Zapata, F. and Roy, R. N. 2007. Utilización de las rocas fosfóricas para una agricultura sostenible. FAO. Internacional de Energía Atómica. Roma, Italia. 73 p.

Zhang, C.; Meng, S.: Li, M. and Zhao, Z. 2016. Genomic identification and expression analysis of the phosphate transporter gene family in poplar. Front Plant Sci. 7:1-14.

Zvobgo, G.; Lwalabawalwalaba, J.; Sagonda, T.; Mutemachani, M. J.; Muhammad, N.; Haider, S. I. and Zhang, G. 2018. Phosphate alleviates arsenate toxicity by altering expression of phosphate transporters in the tolerant barley genotypes. Ecotox. Environ. Safety. 147:832-839. 